\title{
Special issue: Philosophical aspects and implications of the quantum theory of atoms in molecules (QTAIM)
}

\author{
Chérif F. Matta
}

Published online: 22 September 2013

(C) Springer Science+Business Media Dordrecht 2013

It is with great delight that I have accepted the unexpected invitation to edit this two part special issue of Foundations of Chemistry dedicated to the philosophical aspects and implications of the quantum theory of atoms in molecules (QTAIM) (Bader 1990). This theory has been primarily the oeuvre of Richard F. W. Bader (1931-2012), one of his most significant (but not the only significant) contributions to chemistry. Bader's contributions have been summarized in a tribute (Matta et al. 2011) that appeared in a recent Festschrift of The Journal of Physical Chemistry A (2011). This special issue of Foundations is neither a duplication, a complement, or an afterthought to that Festchrift, nor is it intended as another tribute to this great scientist, as deserving as he may be, instead it serves an entirely different purpose. This issue is focused principally on the epistemology and ontology of the science of QTAIM. The sort of questions that are addressed in, or that should emerge from, this issue are of the type: Are atoms in molecules real? What does real mean? Is QTAIM a closed complete theory? Is it a contextual theory? Is QTAIM principally explanatory, accommodative, or predictive and is prediction a necessary condition for an acceptable scientific model or theory or is accommodation sufficient? How fundamental is QTAIM? Is it dependent on the form of the potential in the Hamiltonian? True, some-perhaps even most-of these questions have little or no bearing on the immediate scientific day-to-day practice of QTAIM, but equally true is that they are of paramount importance for the full appreciation of this theory, and for determining its domain of applicability and its position within quantum chemistry and within the broader domain of the physical sciences.

These and similar questions, by remaining largely open, indicate that QTAIM at it deepest level remains to be fully understood, contextualized, and appreciated. This lack of knowledge at the roots, despite of having sparked intense debates, represents no impediment to the wide use and numerous applications of QTAIM in chemistry from stolid state, to material science, to drug design ... This state of affairs is far from being unique in science, actually it is the

Referencing in this article is significantly incomplete, only minimal examples from the literature are cited.

C. F. Matta $(\bowtie)$

Department of Chemistry and Physics, Mount Saint Vincent University, Halifax, NS B3M 2J6, Canada e-mail: cherif.matta@msvu.ca 
norm. Quantum mechanics itself has been successfully applied in all domains of physical science from the days of its inception-even after its completeness has been put into question in 1935 by what became to be know as the EPR-paradox of Einstein, Podolsky, and Rosen (Einstein et al. 1935) — and long before John S. Bell (1928-1990) announced his inequalities and theorem in the sixties of last century that were eventually experimentally tested two decades later by Alain Aspect (1947-) in favour of non-locality. Non-locality, a manifestation of quantum entanglement (or non-separability) and the founding concept of quantum computing (Le Bellac 2006), constitutes a fascinating and probably rare example where a contemporary philosophical debate eventually leading to the development of a technologically promising branch of pure and applied science. ${ }^{1}$ The debates about QTAIM, its meaning, generality, fundamentality, and even whether the very concept of an "atom in a molecule" is a Kantian noumenon or a phenomenon, are and have been vigorous (Parr et al. 2005; Matta and Bader 2006; Matta et al. 2003; Poater et al. 2006; Bader 2002, 2006; Cassam-Chenai and Jayatilaka 2001). Such debates are an unavoidable and healthy "rite of passage" in the life of any new emerging theory before its total assimilation into the established body of human knowledge, after all "no fact is so simple that it is not harder to believe than to doubt at the first presentation" as Lucretius would have said. Sometimes the passion is high, for or against QTAIM, passion that can-at times-and does negatively influence the objectivity and impartiality of the discourse. Can one hope that the debates about the QTAIM will eventually lead to new discoveries following the example of quantum mechanics itself?

Often, QTAIM is criticized for being much more slanted toward accommodation, i.e., the recovery of known facts, in other words it is an explanatory theory rather than a theory that can be used to predict new facts about nature. While one can see why this type of stance may be adopted, I undertake this opportunity to stress that: (1) QTAIM is quite recent, so I urge the proponents of this view to give it time to further evolve and to give the theory the benefit of the doubt, especially that no one has disproved any of its essential elements. (2) Is it really necessary for a theory or a model to be predictive to be useful or its capacity to accommodate is sufficient? In fact, the importance of prediction as contrasted with accommodation is still the subject of a lively debate in the philosophy of science [see for example (Brush 1989, 1993; Scerri and Worrall 2001; White 2003) and the literature cited therein]. (3) Finally, I cite below a few examples from the literature that show how QTAIM is used as a predictive tool and not just as an accommodative theory. Those examples also show that the distinction between prediction and accommodation may not be as sharp as a solid line but that, rather, these are two ends of a continuum that describes the agreement between a theory and Nature.

QTAIM has a coherent self-consistent mathematical structure that conforms with the principles and laws of quantum mechanics. Whether concepts such as the zero-flux interatomic surface which partitions the molecule into separate mononuclear atomic basins $^{2}$ arise naturally from the fundamental principles of quantum mechanics or are additional postulates specific to QTAIM is debatable but is of no consequence on the

\footnotetext{
1 What I mean here is the existence of a clear direct link connecting a philosophical debate to an actual advance in science in recent times. Historically, of course, philosophy and science were more or less merged and one can think of numerous concepts that passed from one to the other, Greek atomism and the modern atomic theory advanced by John Dalton (1766-1844) being an obvious example.

2 In some relatively rare cases, regions of electron density can be bound by zero-flux surfaces around a local maximum but without the existence of any nuclei, these maxima are called non-nuclear attractors (NNA). The basins associated with NNA are "proper open quantum systems" and behave exactly as atoms in molecules in that respect. For that reason, NNA basins are often termed "pseudo-atoms". NNA were first discovered in metals and silicon (Cao et al. 1987; de Vries et al. 1996; Martin-Pendas et al. 1999) and have also been found in systems such as the solvated electron (Taylor et al. 2007).
} 
application of the theory. What is important is that the theory, at the very least, correctly accommodates known facts. But is this theory predictive? And what does it predict?

The short answer is an adamant "yes", it does predict specific thermodynamic, reactivity, and spectroscopic data but it also predicts the overall behavior of atoms and groupings of atoms as they happen to exist in different molecules, i.e., QTAIM predicts data and behavior. I explain.

First, QTAIM defines precisely a number of properties of the atoms, and chemical bonds that link them, in a larger system such as a molecule or a crystal. The properties of atoms and bonds emerge simultaneously and coherently from the topological analysis of the electron density. These properties can be grouped in three broad classes: (1) Atomic properties such as atomic energy, atomic charge, and atomic electric multipoles, are integrated over the (finite) atomic basin (Bader 1990); (2) Integrated interatomic properties such as the delocalization index (Fradera et al. 1999) or the interacting-quantum-atoms (IQA) interaction energy component (Blanco et al. 2005) obtained from integrals over the basins of pairs of atoms; and (3) Bond properties (Bader 1990) which can be further subdivided into those that are locally determined at the bond critical point (BCP) such as the electron density or the energy density evaluated at the $\mathrm{BCP}$, or integrated along the bond path such as the bond path length, or integrated over the interatomic zero-flux surface such as the integrated electron density over the interatomic surface. Atomic properties have been used extensively to accommodate and predict a large array of experimentally verifiable quantities including for example heats of formation (Wiberg et al. 1987), magnetic susceptibility (Keith and Bader 1992), molecular volumes (Bader et al. 1987), polarizability (Bader et al. 1992), Raman intensities (Bader et al. 1992), IR intensities (Gough et al. 2007; Matta and Boyd 2007; César et al. 2005) UV transition probabilities (Bader et al. 2000), physicochemical properties of series of biological molecules (Matta and Arabi 2011), and more. These properties include static and response properties, an example of the latter being the polarizability. The delocalization indices have been used to construct predictive physical models that accurately predict proton-proton (Matta et al. 2002) and fluorine-fluorine (Castillo et al. 2005) NMR coupling constants. A brilliant research program termed the quantum topological molecular similarity (QTMS), developed by Popelier, has demonstrated the exceptional capability of bond properties to encode and summarize molecular properties and predict not only drug activity but even pinpoint the location of the active site within the molecule (Popelier 1999; O'Brien and Popelier 2001).

Besides the prediction of specific properties, the atoms of QTAIM emulate the observed behavior of atoms in real molecules, that is, they have a paralleling transferability and additivity of their properties, both long known in thermochemistry. During years as one of the graduate students in Richard Bader's group, I have heard him numerous times express great interest and admiration for Sidney W. Benson's (1918-2011) work on group additivity schemes, the use of which is now widespread in physicochemical research and industry. The success of this empirical approach to predict the properties of large and often complex molecules from the contributions of fragments rests on the simultaneous transferability and additivity of the properties of atoms and groupings of atoms. The atoms of QTAIM have been shown numerous times to exhibit corresponding transferability and additivity, and to use one of Bader's favourite phrases, the "atoms of theory" behave in an identical manner as the atoms of the laboratory.

Additivity is a clear concept, that the sum of the atomic (or group) properties yields the corresponding molecular properties, a necessary consequence of the exhaustive partitioning of the molecular physical three-dimensional space by the zero-flux surfaces. But how does transferability work? A close examination reveals two types of transferability: 
Direct transferability and compensatory transferability, both types being crucial for the emulation of Benson's group additivity schemes by the atoms of theory.

Direct transferability is the approximate constancy of the atomic properties of the same group in different molecules, while compensatory transferability means that the change in one atom or group is canceled by a nearly equal and opposite change in another atom or group in the molecule, often-but no always-its bonded neighbor. Cortés-Guzmán and Bader (2004) close their paper with the remarkable statement that clarifies further the latter concept:

There appears to be a Le Châtelier principle at work-one that states that two open systems brought into contact respond in such a way as to minimize the overall changes in form and energy, resulting in many cases in a conservation of energy.

I do not know if Bader and Benson have ever met in real life but-remarkably-their obituaries appear side-by-side in the same page of Chemical \& Engineering News (the issue of 26 March 2012, page 58).

The transferability and additivity of the atoms defined by QTAIM have been used extensively to reconstruct the properties of large molecules from smaller fragments [See for example: (Chang and Bader 1992; Sukumar and Breneman 2007; Scheins et al. 2005)]. If such reconstruction of the properties of large molecules from composing atoms and groups stored in a database, with chemical accuracy, does not constitute prediction, how would that be characterized? From the "atoms of theory" thus, indeed, not only specific observational chemical data can be predicted but also the behavior of the atoms of the laboratory is closely shadowed within chemical accuracy as well. We can reasonably conclude that accumulated empirical evidence demonstrates that QTAIM is both accommodative and predictive.

Since the theory affords predictions that can be tested by experiment then QTAIM is a falsifiable theory in Popper's (1959) sense. Furthermore, the theory has never been falsified, despite the occasional claims based on either improper application, lack of understanding, or over-interpretation. In almost all cases, these occasional claims of falsification have been rebutted completely, definitively, and forcefully by Bader and others both in print and at conferences.

To sum-up, QTAIM is expressed as a coherent mathematical structure that conforms with the principles and laws of quantum mechanics, possibly with additional postulates ( $a$ debatable but inconsequential issue); it accommodates known facts and leads to verifiable predictions, that is, it is falsifiable; and has never been falsified.

Before leaving these introductory comments it is important to note that QTAIM is not a closed theory and that it is still being extended and expanded beyond its original reach and scope. For example, James Anderson and Paul Ayers have recently shown the possibility of extending QTAIM and the zero-flux condition to electron densities that incorporate relativistic effects (Anderson and Ayers 2011) and, simultaneously, Shant Shahbazian and coworkers have demonstrated that the concepts of QTAIM survive even beyond the BornOppenheimer (BO) approximation where protons are no longer treated as clamped classical particles but are truly delocalized quantum particles and thus the analysis of the electron density is supplanted in this approach by that of the joint electron-proton charge density (Goli and Shahbazian 2011). Mark Eberhart and Travis Jones have developed the concept of "irreducible bundle" that partitions the atomic basin itself into proper open quantum systems bounded by local zero-flux surfaces (in this case, and unlike interatomic surfaces, the partitioning surfaces, also not crossed by the gradient vectors, reach and partition the atomic nuclei) (Jones et al. 2012; Jones and Eberhart 2009, 2010) to predict the strength 
and tensile properties of metallic alloys. These are the same surfaces used previously to attack QTAIM (Cassam-Chenai and Jayatilaka 2001; Bader 2002). QTAIM is thus still open for innovation and for new exciting developments that push its frontiers.

It is fitting to start this two part special issue with Richard Bader's last (and posthumous) publication titled "Atoms in molecules as non-overlapping, bounded, space-filling open quantum systems". This paper is a revised and updated version of an old manuscript that Richard and I originally titled "Sea Change", and that was rejected in 2001 by the Journal of Chemical Education. The manuscript was totally forgotten for a decade, but the death of Richard prompted me to submit it for publication in this special issue as it constitutes a part, even if small, of his legacy. The paper emphasizes that QTAIM is built on analyzing "real measurable fields" and that the theory provides an intelligible link between experiment and theory as opposed to models based on arbitrary choices and nonmeasurable quantities.

Part I then proceeds with the provocative question The two faces of chemistry: can they be reconciled? posed by Mark Eberhart and Travis Jones. Shant Shahbazian then summarizes and reviews some aspects of his two-component (non-BO) QTAIM in his essay "Beyond the orthodox QTAIM: motivations, current status, prospects and challenges". The relationship between atoms in a molecule and each other and between each atom and the whole they compose is the topic of "The atom in a molecule as a mereological construct in chemistry" by Nagamani Sukumar. In "Austere quantum mechanics as a reductive basis for chemistry", Hinne Hettema provides argumentation for considering QTAIM as a regulative and constructive theory of chemistry. Finally, and concluding the first Part of this issue is a critique of some technical aspects of Hettema's paper by Shahbazian followed by Hettema's response.

The second part of this issue opens with "Atoms and bonds in molecules and chemical explanations" an article in which Mauro Causá, Andreas Savin, and Bernard Silvi explain how QTAIM, the electron localization function (ELF) and the maximum probability domains (MPD) all contribute at linking the autonomous science of chemistry with physics. This is followed by Roman Nalewajski's "Entropic concepts in electronic structure theory" in which he explores the contribution of quantities related to and derived from Shannon's entropy (information theoretic) to atoms in molecules and in the quantitative description of their interaction by such quantities as bond orders. The special issue ends with a thought provoking essay by Shant Shabazian titled "What does shape a topological atom?" followed by a letter to the editor by the same author titled "Are there "really" atoms in molecules?"

I thank all the authors of this special issue and the anonymous reviewers who contributed at improving the quality of the papers considerably. In preparing this special issue, I have benefitted considerably from discussions with Paul W. Ayers, Richard F. W. Bader, Ian Hamilton, Samantha Jenkins, Todd A. Keith, Claude Lecomte, Jean Pierre-Llored, Lou Massa, SeyedAbdolreza Sadjadi, Eric Scerri, Paul von-Ragué Schleyer, Eugen Schwarz, Shant Shahbazian, Shahin Sowlati-Hashjin, and Matthew Timm—all of whom I warmly thank. I thank the Natural Sciences and Engineering Research Council of Canada (NSERC), Canada Foundation for Innovation (CFI), and Mount Saint Vincent University, for their financial support.

\section{References}

Richard F. W. Bader Festschrift. J. Phys. Chem. A 111(45), 12427-13209 (2011)

Anderson, J.S.M., Ayers, P.W.: Quantum theory of atoms in molecules: results for the SR-ZORA Hamiltonian. J. Phys. Chem. A 115, 13001-13006 (2011) 
Bader, R.F.W.: Atoms in Molecules: A Quantum Theory. Oxford University Press, Oxford, UK (1990)

Bader, R.F.W.: A comment on "Some fundamental problems with zero-flux partitioning of electron densities". Theor. Chem. Acc. 107, 381-382 (2002)

Bader, R.F.W.: Pauli repulsions exist only in the eye of the beholder. Chem. Eur. J. 12, 2896-2901 (2006)

Bader, R.F.W., Bayles, D., Heard, G.L.: Properties of atoms in molecules: transition probabilities. J. Chem. Phys. 112, 10095-10105 (2000)

Bader, R.F.W., Carroll, M.T., Cheeseman, J.R., Chang, C.: Properties of atoms in molecules: atomic volumes. J. Am. Chem. Soc. 109, $7968-7979$ (1987)

Bader, R.F.W., Keith, T.A., Gough, K.M., Laidig, K.E.: Properties of atoms in molecules: additivity and transferability of group polarizabilities. Mol. Phys. 75, 1167-1189 (1992)

Blanco, M.A., Martin Pendas, A., Francisco, E.: Interacting quantum atoms: a correlated energy decomposition scheme based on the quantum theory of atoms in molecules. J. Chem. Theor. Comput. 1, 1096-1109 (2005)

Brush, S.G.: Prediction and theory evaluation: the case of light bending. Science 246, 1124-1129 (1989)

Brush, S.G.: Prediction and theory evaluation: subatomic particles. Riv. Stor. Scienz. Ser. II 1, 47-152 (1993)

Cao, W.L., Gatti, C., MacDougall, P.J., Bader, R.F.W.: On the presence of non-nuclear attractors in the charge distributions of Li and Na clusters. Chem. Phys. Lett. 141, 380-385 (1987)

Cassam-Chenai, P., Jayatilaka, D.: Some fundamental problems with zero flux partitioning of electron densities. Theor. Chem. Acc. 105, 213-218 (2001)

Castillo, N., Matta, C.F., Boyd, R.J.: Fluorine-fluorine spin-spin coupling constants: correlations with the delocalization index and with the internuclear separation. J. Chem. Inf. Mod. 45, 354-359 (2005)

Chang, C., Bader, R.F.W.: Theoretical construction of a polypeptide. J. Phys. Chem. 96, 1654-1662 (1992)

Cortés-Guzmán, F., Bader, R.F.W.: Role of functional groups in linear regression analysis of molecular properties. J. Phys. Org. Chem. 17, 95-99 (2004)

César, P.H., Faria, S.H.D.M., a Jr, J.V., Haiduke, R.L.A., Bruns, R.E.: A charge-charge flux-dipole flux decomposition of the dipole moment derivatives and infrared intensities of the $\mathrm{AB}_{3}(\mathrm{~A}=\mathrm{N}, \mathrm{P} ; \mathrm{B}=\mathrm{H}$, F) molecules. Chem. Phys. 317, 35-42 (2005)

de Vries, R.Y., Briels, W.J., Feil, D., te Velde, G., Baerends, E.J.: Charge density study with maximum entropy method on model data of silicon. A search for non-nuclear attractors. Can. J. Chem. 74, 1054-1058 (1996)

Einstein, A., Podolsky, B., Rosen, N.: Can quantum-mechanical description of physical reality be considered complete? Phys. Rev. 47, 777-780 (1935)

Fradera, X., Austen, M.A., Bader, R.F.W.: The Lewis model and beyond. J. Phys. Chem. A 103, 304-314 (1999)

Goli, M., Shahbazian, S.: Atoms in molecules: beyond Born-Oppenheimer paradigm. Theor. Chem. Acc. 129, 235-245 (2011)

Gough, K.M., Dawes, R., Dwyer, J.R., Welshman, T.L.: QTAIM analysis of Raman scattering intensities: insights into the relationship between molecular structure and electronic charge flow. In: Matta, C.F., Boyd, R.J. (eds.) The Quantum Theory of Atoms in Molecules: From Solid State to DNA and Drug Design, pp. 95-120. Wiley-VCH, Weinheim (2007)

Jones, T.E., Eberhart, M.E.: The irreducible bundle: Further structure in the kinetic energy distribution. J. Chem. Phys. 130, 204108 (2009)

Jones, T.E., Eberhart, M.E.: The bond bundle in open systems. Int. J. Quantum Chem. 110, 1500-1505 (2010)

Jones, T.E., Eberhart, M.E., Imlay, S., Mackey, C., Olson, G.B.: Better alloys with quantum design. Phys. Rev. Lett. 109, 125506 (2012)

Keith, T.A., Bader, R.F.W.: Calculation of magnetic response properties using atoms in molecules. Chem. Phys. Lett. 194, 1-8 (1992)

Le Bellac, M.: A Short Introduction to Quantum Information and Quantum Computation. Cambridge University Press, Cambridge (2006)

Martin-Pendas, A., Blanco, M.A., Costales, A., Mori-Sanchez, P., Luana, V.: Non-nuclear maxima of the electron density. Phys. Rev. Lett. 83, 1930-1933 (1999)

Matta, C.F., Arabi, A.A.: Electron-density descriptors as predictors in quantitative structure-activity/property relationships and drug design. Future Med. Chem. 3, 969-994 (2011)

Matta, C.F., Bader, R.F.W.: An experimentalist's reply to "what is an atom in a molecule?". J. Phys. Chem. A 110, 6365-6371 (2006)

Matta, C.F., Hernández-Trujillo, J., Bader, R.F.W.: Proton spin-spin coupling and electron delocalisation. J. Phys. Chem. A 106, 7369-7375 (2002) 
Matta, C.F., Hernández-Trujillo, J., Tang, T.H., Bader, R.F.W.: Hydrogen-hydrogen bonding: a stabilizing interaction in molecules and crystals. Chem. Eur. J. 9, 1940-1951 (2003)

Matta, C.F., Massa, L., Keith, T.A.: Richard F. W. Bader: a true pioneer. J. Phys. Chem. A 115, 12427-12431 (2011)

O’Brien, S.E., Popelier, P.L.A.: Quantum molecular similarity. 3. QTMS descriptors. J. Chem. Inf. Comput. Sci. 41, 764-775 (2001)

Parr, R.G., Ayers, P.W., Nalewajski, R.F.: What is an atom in a molecule. J. Phys. Chem. A 109, 3957-3959 (2005)

Poater, J., Solà, M., Bickelhaupt, F.M.: A model of the chemical bond must be rooted in quantum mechanics, provide insight, and possess predictive power. Chem. Eur. J. 12, 2902-2905 (2006)

Popelier, P.L.A.: Quantum molecular similarity. 1. BCP space. J. Phys. Chem. A 103, 2883-2890 (1999)

Popper, K.: The Logic of Scientific Discovery. Routledge, London (1959)

Scerri, E.R., Worrall, J.: Prediction and the periodic table. Stud. Hist. Philos. Sci. 32, 407-452 (2001)

Scheins, S., Messerschmidt, M., Luger, P.: Submolecular partitioning of morphine hydrate based on its experimental charge density at $25 \mathrm{~K}$. Acta Cryst. B 61, 443-448 (2005)

Sukumar, N., Breneman, C.M.: QTAIM in drug discovery and protein modeling. In: Matta, C.F., Boyd, R.J. (eds.) The Quantum Theory of Atoms in Molecules: From Solid State to DNA and Drug Design, pp. 473-498. Wiley-VCH, Weinheim (2007)

Taylor, A., Matta, C.F., Boyd, R.J.: The hydrated electron as a pseudo-atom in cavity-bound water clusters. J. Chem. Theor. Comput. 3, 1054-1063 (2007)

White, R.: The epistemic advantage of prediction over accommodation. Mind 112, 653-683 (2003)

Wiberg, K.B., Bader, R.F.W., Lau, C.D.H.: Theoretical analysis of hydrocarbons properties. 2. Additivity of group properties and the origin of strain energy. J. Am. Chem. Soc. 109, 1001-1012 (1987) 